\title{
Trastorno Antisocial de la Personalidad: Epidemiología, Criterios de Diagnóstico y Tratamiento Psicoterapéutico
}

\author{
Antisocial Personality Disorder: Epidemiology, Diagnostic Criteria and Psychotherapeutic \\ Treatment
}

\author{
Barraza-Sánchez, $B^{l}$; Iglesias-Hermenegildo $A^{2}$; Serrano-Ruíz José Jesús ${ }^{3}$
}

\begin{abstract}
:
The antisocial personality disorder (ASPD) was the first personality disorder studied and described by mental health professionals. Some of its clinical characteristics are lying, manipulating, showing narcissistic features (1) and an antisocial behavior, that is, showing disregard for the rights of others, and for social regulations, and displaying aggressive and destructive behavior.

According to data obtained by the National Survey of psychiatric Epidemiology, in Mexico, the dissocial disorder is present in about 10.3 per cent of male population and in 2.3 percent of female population. This pathology appears during adolescence and if not treated in an appropriate and expedient way, it is considered to be a precedent of the antisocial personality disorder (2).

This paper addresses the etiology, including biological, psychological, neuropsychological and sociocultural factors that intervene in the etiology and development of the antisocial personality disorder.

The DSM-V (2013) diagnostic criteria of the American Psychiatric Association and the ICD diagnostic criteria of the International Classification of Diseases of the World Health Organization are included in this paper. In addition to this, a description of the clinical characteristics from a psychodynamic perspective is provided.
\end{abstract}

Treatment of the antisocial personality disorder includes pharmacological and social treatment, as well as psychotherapy, particularly expressive and supportive psychotherapy.

\section{Keywords:}

Antisocial personality disorder, clinical characteristics, psychodiagnostic and psychotherapeutic treatment.

\section{Resumen:}

El trastorno antisocial de la personalidad [TAP] fue el primer trastorno de personalidad que se abordó por profesionales de la salud mental y del que se proporcionó una descripción de sus características clínicas, entre las que se encuentran mentir, ser manipulador, mostrar rasgos narcisistas (1), hay un comportamiento antisocial, es decir, se violan los derechos de otras personas y las reglas y normas sociales por medio de comportamientos agresivos y destructivos. Según datos obtenidos en la Encuesta Nacional de Epidemiología Psiquiátrica en México, el trastorno disocial se presenta en alrededor del 10.3 por ciento de los hombres mientras que en las mujeres se manifiesta en un 2.3 por ciento de la población a nivel general; esta patología inicia durante la adolescencia y de no ser atendido de forma adecuada y oportuna se considera como un antecedente para el desarrollo del trastorno antisocial de la personalidad (2).Se aborda la etiología, incluyendo factores biológicos, psicológicos, neuropsicológicos y socioculturales que intervienen en el inicio y desarrollo del trastorno antisocial de la personalidad. Se incluyen los criterios diagnósticos basados en el DSM-V (2014) de la Asociación Psiquiátrica Americana y del CIE-10 (1999) de la Organización mundial de la salud, así mismo se da una descripción de características clínicas psicodinámicas. El tratamiento del trastorno antisocial de la personalidad incluye lo

\footnotetext{
${ }^{1}$ Alumno de la maestría en Salud Pública. Universidad Autònoma del Estado de Hidalgo, Instituto de Ciencias de la Salud, Pachuca, Hidalgo. México. Email: brenbrrz@hotmail.com

2 Autor de Correspondencia. Profesora Investigador de Tiempo Completo. Universidad Autònoma del Estado de Hidalgo, Instituto de Ciencias de la Salud, Pachuca, Hidalgo. México. ORCID: 0000-0002-4922-3884. Email: an.iglesias13@ gmail.com.

${ }^{3}$ Profesor por asignatura del área académica de Psicología y Gerontología. Universidad Autònoma del Estado de Hidalgo, Instituto de 
farmacológico, social y psicoterapéutico y de manera específica se aborda la psicoterapia expresiva y de apoyo para el tratamiento de este trastorno.

\section{Palabras Clave:}

Trastorno antisocial de la personalidad, características clínicas, Psicodiagnóstico y tratamiento psicoterapéutico.

\section{Epidemiología}

La epidemiología ha hecho contribuciones importantes para el conocimiento de la distribución, frecuencia y factores determinantes de los trastornos mentales en general, pero en particular ha sido muy relevante para el desarrollo de la psicopatología clínica. Es en el campo específico de la psicopatología donde la epidemiología de los trastornos mentales ha hecho las contribuciones más notorias, señalando al clínico las limitaciones de los casos que atienden en los servicios especializados, poniéndolos en relación con la población de donde provienen y completando el cuadro clínico de algunos padecimientos mentales (3).

Los hallazgos de las Encuestas Mundiales de Salud Mental de diferentes países muestran que los trastornos psiquiátricos consistentemente inician en las primeras décadas de la vida (4). Sin embargo, hay una escasez de datos epidemiológicos en población adolescente, especialmente en países en vías de desarrollo como el nuestro para guiar las políticas de salud pública.

Entre los trastornos más frecuentes en la adolescencia se encuentran los trastornos de conducta y de ansiedad, siendo estos los que tienen las edades de inicio más tempranas. (5)

Una investigación realizada por Fazel y Danesh (6) en la que se analizaron 62 estudios psiquiátricos de poblaciones reclusas de 14 países de occidente, se encontró una incidencia en varones del 65 por ciento respecto a los trastornos de personalidad, en el que un 47 por ciento corresponde al trastorno antisocial de la personalidad y un 3.7 por ciento hace referencia a estados psicopáticos, mientras que el 10 por ciento tiene que ver con el trastorno depresivo. En cuanto a las mujeres los trastornos de personalidad como la psicopatía era ligeramente menor en relación a los varones, mientras que el [TAP] tiene una prevalencia mucho menor con un 21 por ciento de los casos, sin embargo, la depresión aumenta presentándose en el 12 por ciento de los casos.

Aunque la mayoría de los estudios internacionales no han encontrado diferencias consistentes respecto al sexo en la prevalencia general de los trastornos de personalidad, algunas investigaciones sugieren diferentes proporciones respecto al sexo para trastornos de personalidad específicos, como en el caso del trastorno antisocial donde se presenta una menor prevalencia en mujeres, pero una mayor prevalencia de trastornos por dependencia, de evitación y el trastorno de personalidad paranoide.

\section{Nacional}

La Encuesta Nacional de Epidemiología Psiquiátrica (ENEP), realizada durante el 2001 y el 2002 proporcionó las primeras estimaciones representativas a nivel nacional de los trastornos mentales en la población mexicana. Además, la ENEP, demuestra que los trastornos psiquiátricos se inician durante las primeras décadas de vida, lo que es consistente con los datos epidemiológicos a nivel mundial. De acuerdo a la Encuesta Nacional de Epidemiología psiquiátrica, en México un 10.3 por ciento de los varones presentan un trastorno disocial, antecedente del trastorno antisocial de la personalidad, mientras que en las mujeres se manifiesta en un 2.3 por ciento (2).

Otro estudio realizado en México en el año 2005 fue la Encuesta de Salud Mental en la población adolescente de la Ciudad de México y el área metropolitana como parte de la iniciativa de la Organización Mundial de la Salud Mental, este estudio brindó datos importantes en cuanto a epidemiología psiquiátrica de niños y adolescentes se refiere. Entre los principales resultados se encontró que los trastornos individuales más frecuentes en ambos sexos fueron las fobias específicas y la fobia social, en el caso de los varones, el trastorno que sigue a las fobias es el trastorno negativista desafiante, seguido por el abuso de alcohol y el trastorno de la personalidad disocial. Además, se encontró que las mujeres presentan un número mayor de trastornos psicológicos y una mayor prevalencia sobre todo en trastornos de ansiedad, cualquier trastorno de ánimo y cualquier trastorno de impulsividad (aunque este último se debe al trastorno negativista desafiante y no al trastorno disocial o por déficit de atención) (7).

Otro dato importante derivado de esta encuesta en relación al tema que nos ocupa es la edad de inicio de los trastornos de impulsividad, ya que los hombres tienen edades de inicio más tempranas que las mujeres. Así mismo, se encontró que las diferencias por sexo en los trastornos impulsivos no se deben a diferencias en la prevalencia del déficit de atención o del trastorno disocial, sino a la presencia del trastorno negativista desafiante en las y los adolescentes (7).

En la Encuesta Nacional de la Juventud realizada en 2005, en la ciudad de México, se encontró que el 51.3 por ciento de los encuestados cumplieron con los criterios diagnósticos de un trastorno mental alguna vez en vida, la edad de inicio de los trastornos afectivos y de impulsividad donde se ubica el trastorno disocial, oscila entre los 11 y 12 años de edad, y la prevalencia es del 4.1 por ciento en adolescentes (5). 
La edad en que puede comenzar a presentarse el trastorno disocial en hombres oscila entre los 10 y 13 años y en mujeres se presenta entre los 14 y 16 años (8). Mientras que la prevalencia del trastorno de personalidad antisocial en varones es de uno a seis por ciento en la población en general, es observable en centros de atención a la salud mental y sobre todo en el sistema penitenciario ya que se calcula que del total de esta población dicho trastorno está presente en aproximadamente el 80 por ciento (9).

Respecto a algunas de las características sociales y psicológicasque presentan los pacientes con este trastorno se evidenció una lista de 23 situaciones traumáticas, el 69 por ciento de los jóvenes experimentó violencia intrafamiliar con un 19 por ciento, seguido de amenazas o víctima de asalto con un arma con el 17 por ciento, 13 por ciento sufrió maltrato físico por parte de los cuidadores, el 5 por ciento fue víctima de abuso sexual y el 1 por ciento notifico violación (5).

En relación con la mortalidad de los adolescentes con trastorno antisocial de la personalidad encontramos que es de 10 a 14 veces mayor que la población que no padece este trastorno, debido a que violan la ley cometiendo delitos violentos como asalto y allanamiento, mientras que las mujeres adolescentes diagnosticadas con [TAP] tienen diez veces más propensión a morir que los hombres con dicho trastorno debido a que se ven relacionadas con consumo de drogas y prostitución (10).

Las cifras anteriores son alarmantes y permiten a los profesionales de la salud mental observar la necesidad de implementar estrategias que permitan una adecuada y oportuna atención de los padecimientos mentales, además de generar una reestructuración de las instituciones de salud mental de nuestro país, para mejorar los servicios y con ello la calidad de vida de los pacientes con trastornos mentales, cómo lo son los pacientes con TAP.

\section{Estatal}

El estado de Hidalgo no tiene un panorama distinto al mundial y nacional en lo que a datos epidemiológicos se refiere, sobretodo en relación a los trastornos de personalidad y específicamente al trastorno antisocial.

A través del Modelo Hidalgo de Salud Mental, el Estado de Hidalgo se encuentra como un referente nacional en cuanto a prevención y atención de la salud mental se refiere. Incluso en el año 2013 expertos de la Organización Panamericana de la Salud [OPS] (11) y Organización Mundial de la Salud [OMS] (12) conocieron el modelo Miguel Hidalgo de atención en Salud Mental de la Secretaría de Salud de Hidalgo [SSH], a fin de recabar experiencias exitosas en la implementación de éste, para retomarlas y replicarlas en otros países (13).

Algunos datos, que proporcionan un acercamiento a la epidemiología de los trastornos mentales en Hidalgo, se describen a continuación.
En el marco de las primeras jornadas estatales de enfermería "Calidad de enfermería psiquiátrica y salud mental, compartiendo conocimiento y experiencia" realizada en el 2016, en el Hospital Villa Ocaranza; el secretario de salud de Hidalgo Marco Antonio Escamilla Acosta enfatizó que a pesar de que los trastornos mentales son un tema rezagado por la misma sociedad, actualmente representan un problema de salud pública pues tan solo en México, los padecimientos de tipo psiquiátrico, psicológico o neurológico afectan a cerca de 16 millones de personas. En 2016 la Secretaría de Salud de Hidalgo a través del director del Hospital Psiquiátrico Villa Ocaranza, destacó que dentro de las primeras causas de demanda de salud mental se encuentran patologías como la esquizofrenia, trastornos de personalidad, los trastornos del estado de ánimo, trastornos por consumo de sustancias psicoactivas y ansiedad (14).

En un estudio realizado en 2015 por Centro Estatal para la Atención Integral de las Adicciones de Pachuca de Soto, sobre funcionalidad familiar y patología dual en usuarios residenciales del Hidalgo, se encontró como parte de los resultados preliminares que el trastorno psiquiátrico más común en este tipo de pacientes es la distimia, seguido del trastorno depresivo mayor, trastorno de ansiedad generalizada y trastorno de la conducta de tipo negativista desafiante en sujetos menores de 18 años. Mientras que los trastornos de personalidad más comunes fueron el trastorno de inestabilidad emocional de la personalidad de tipo impulsivo y tipo límite y el trastorno antisocial de la personalidad (15).

En un estudio coordinado por la Dra. Azaola (2015) en colaboración con la UNICEF, se realizó un diagnóstico de las y los adolescentes que cometen delitos graves en México. Entre los objetivos de este estudio, estuvo elaborar un diagnóstico sobre la situación de las y los adolescentes en conflicto con la ley en cuatro entidades y entre ellas se encontraba el Estado de Hidalgo. Si bien los resultados no reportan datos estadísticos relacionados con los trastornos mentales que presentan los adolescentes que se encuentran en una situación de reclusión, si se reportan datos que ofrecen un panorama sobre algunas conductas y aspectos emocionales sobre esta población que como la literatura refiere presentan el trastorno antisocial y disocial. Los jóvenes internos refieren que el trato por parte del personal (psicólogos, pedagogos, abogados, cuerpo policiaco etc.) es según el 81 por ciento bueno, 14 por ciento lo considera regular y 5 por ciento piensa que es malo, únicamente el 21 por ciento del total de los adolescentes que participó en el estudio los adolescentes acude a terapias con psicólogos dentro del centro de internamiento (16)

Estos datos, permiten identificar que no se les ha dado la atención que merecen y a pesar de los avances en esta materia aún existe un gran desconocimiento sobre las enfermedades mentales y su tratamiento. 


\section{Historia del Trastorno Antisocial de la Personalidad}

Gran parte de la literatura existente emplea los términos de psicópata, sociópata o locura moral como sinónimos para referirse a las características clínicas y los criterios diagnósticos que actualmente definen al trastorno antisocial de la personalidad, esta terminología es utilizada por psicólogos y psiquiatras, quienes emplean los términos según los métodos o la población a estudiar, por lo que puede considerarse que en la mayoría de los casos se refieren a lo mismo (17). Recientemente el término psicópata se ha dejado de utilizar, sobre todo en las clasificaciones clínicas de trastornos como el DMS y el CIE-10 y se ha optado por utilizar términos como trastorno antisocial de la personalidad y/o trastorno disocial (6).

Es así que a lo largo de la historia ha habido una evaluación sobre los términos utilizados para denominar a lo que hoy se conoce como trastorno antisocial de la personalidad por lo que a continuación se presenta un esquema sobre esta evolución.

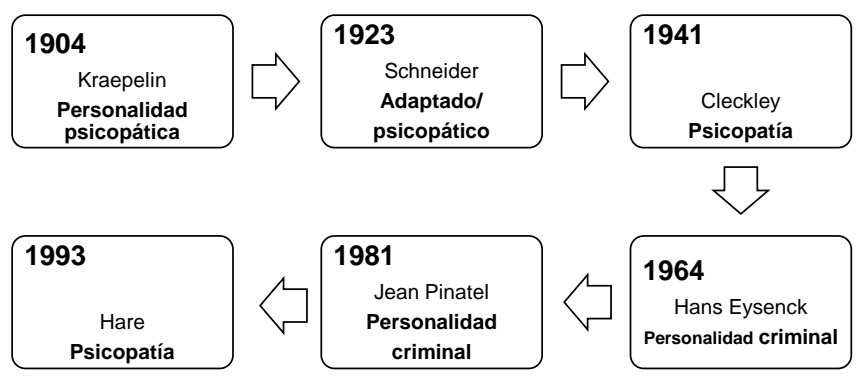

En cuanto al DSM V (18) también se han realizado cambios respecto a la nomenclatura del trastorno antisocial de la personalidad, como se muestra en el siguiente esquema.

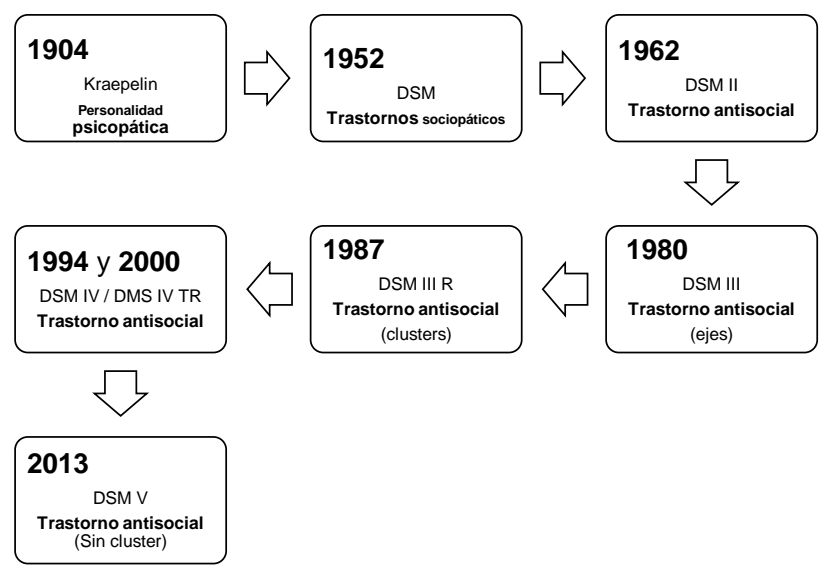

\section{Etiología del Trastorno Antisocial de la Personalidad}

\section{Factores biológicos y neuropsicológicos}

Se considera que las causas que dan pie a la presencia del trastorno antisocial son de origen biológico y psicosocial, existen estudios que han demostrado una significativa relación entre la genética y el contexto, así, cuando en un sujeto la predisposición genética se combina con una crianza con aspectos negativos, es decir, violencia, rechazo, maltrato durante la infancia, generan un incremento en las probabilidades de que la conducta antisocial ocurra (9).

Según las investigaciones biopsicológicas existe una relación entre la conducta antisocial y factores biológicos, por ejemplo, los instintos de supervivencia y algunos procesos de orden bioquímico como la testosterona, adrenalina y noradrenalina, además de la serotonina, las disfunciones electroencefalográficas, alteraciones cromosómicas, la alta impulsividad, así como la influencia genética (19).

En un estudio realizado por García \& Ostrosky-Solis (20) con reclusos de una prisión federal mexicana, se encontró que los sujetos que fueron diagnosticados con psicopatía, presentan alteraciones en las funciones frontales y ejecutivas por lo que existen deficiencias en las funciones orbifrontales y dorsolaterales. Siendo la corteza orbital una estructura primaria de regulación de la inhibición, el razonamiento moral y toma de decisiones, de modo que son dichas habilidades las más significativas a la hora de manifestar conductas violentas, por lo que las alteraciones en el funcionamiento orbifrontal se relacionan con la presencia del trastorno antisocial de la personalidad.

Dentro del modelo neuropsicológico se han realizado algunos exámenes que señalan que los niños y adolescentes que presentan trastornos de conducta muestran dificultades en el funcionamiento del lóbulo frontal, lo que interfiere con la capacidad para evitar riesgos, así como planificar y aprender de las experiencias negativas (15) (21). Además el modelo neuropsicológico hace referencia a la relación existente entre el funcionamiento de las estructuras neurofisiologícas y las funciones psicológicas, ya que el hipotálamo se relaciona con la motivación y la emoción, destacando la influencia de las estructuras cerebrales límbicas, la amígdala y el septum en la presencia de la conducta delictiva (19).

Sin duda los factores biológicos son determinantes en la presencia del trastorno antisocial de la personalidad, y deben ser tomados en cuenta por el clínico a la hora de realizar una evaluación y posterior diagnóstico, ya que de ellos depende una parte del avance psicoterapéutico del paciente, pues en algunos casos es necesario complementar el tratamiento psicológico con atención psiquiátrica. 


\section{Factores Psicosociales}

Para considerar que un sujeto padece un trastorno antisocial de la personalidad se deben tomar en cuenta factores individuales como el temperamento, el carácter, la impulsividad y las emociones, por mencionar algunos.

La respuesta emotiva, así como el grado de autorregulación del sujeto pueden originar la interiorización y/o exteriorización de problemas durante la infancia que pueden llevar a trastornos de conducta, que continúen hasta la adolescencia generando la presencia del TAP durante los inicios de la adultez (22).

En cuanto a las características de personalidad, se considera que responden a un comportamiento general de desprecio y violación de los derechos de otras personas, acompañada de indiferencia y falta de remordimiento al infringir daños a terceros, inexistencia de sentimientos de empatía o respeto, estas se presentan desde la infancia y continúan en la adolescencia; si permanecen hasta la edad adulta, puede hablarse de que se trata del trastorno antisocial de la personalidad, aunque se deben evaluar diversos criterios antes de realizar un diagnóstico (23).

También deben considerarse los factores de riesgo de las conductas antisociales, que hacen alusión a aquellas características individuales y ambientales que incrementan la probabilidad de ejecución y mantenimiento de dichas conductas (21). La criminalidad de los padres combinada con un bajo estatus social, de igual forma que si la madre o el padre tiene problemas de alcoholismo o adicción a alguna droga son considerados como factores de riesgo para que los adolescentes desarrollen el TAP (17).

Se ha comprobado que existe una relación entre los sucesos traumáticos que vive un sujeto, sobre todo durante los primeros años de vida, como el abuso físico y/o emocional y la presencia de trastornos de la personalidad, siendo más significativa la relación entre el abuso físico y la presencia del trastorno antisocial (6). Los menores que han sido criados en un núcleo familiar de riesgo manifiestan mayor número de comportamientos antisociales además de una falta de adaptación en todos los niveles, muestran desajustes en habilidades sociales, estrategias de afrontamiento, autoconcepto, así como en el estado clínico del sujeto (24).

Desde el punto de vista psicoanalítico se describen algunas causas por las que se desarrollan las estructuras de personalidad antisociales, entre ellas se encuentran las fallas del vínculo madre/hijo durante los primeros años de vida (25).

Singer (26) realizó un estudio con delincuentes de clase media que presentan conductas antisociales, sin llegar a ser sociópatas graves. Toma cinco rutas de investigación que representan los puntos de interés de diferentes autores en momentos distintos: desórdenes pulsionales y formación de fantasía, desórdenes caracterológicos y del yo, defectos del superyó, desórdenes de interacción diádica y desórdenes en la interacción familiar.

Winnicott (27) realizó varios estudios desde el psicoanálisis para conocer más respecto a las personalidades antisociales, sobre todo durante la niñez y la adolescencia; encontró respecto a la etiología, que un niño que no tiene un sostén familiar adecuado, refiriéndose con ello a la carencia o ausencia de vínculos y afectos, fenómeno al que denomina deprivación, y el cual genera ansiedades y confusiones que sobrepasan al sujeto, pretende anularlas mediante actos delictivos, por lo que la patología según este autor, tiene su origen en el ambiente social o cultural y puede presentarse a cualquier edad.

\section{Criterios Diagnósticos del Trastorno Antisocial de la Personalidad}

Para realizar el diagnóstico del TAP se deben tomar en cuenta datos cualitativos y cuantitativos, así como valorar el estilo de vida del paciente, comenzando desde la niñez - adolescencia, ya que es cuando comienzan a presentarse los problemas de conducta, por ejemplo, robo, impulsividad, huidas de casa, así como antecedentes de incumplimiento en la escuela o el trabajo. Otras características importantes de la personalidad antisocial se refieren a la incapacidad de posponer el placer inmediato o la gratificación de impulsos, además a estos pacientes se les dificulta establecer relaciones cercanas con otros sujetos, un rasgo característico es la ausencia de culpa y ansiedad por la comisión de actos antisociales (7).

La Asociación Psiquiátrica Americana en el Manual Diagnóstico y Estadístico de los trastornos Mentales [DSM V] (18) ubica al trastorno antisocial de la personalidad dentro del grupo $B$, junto con los trastornos limítrofe, histriónico y narcisista. Menciona que el TAP comienza durante la niñez o adolescencia temprana, con un comportamiento irresponsable y con frecuencia criminal, junto con ausentismo escolar, huidas del hogar, peleas, crueldad, destrucción, falsedad y robo. Ya en la adultez, además del comportamiento criminal actúan de forma irresponsable o impulsiva, sin mostrar remordimiento alguno por sus acciones. Los criterios para el diagnóstico que propone el DSM V (18) para el TAP se refieren en primer lugar a: A) un patrón dominante de falta de atención y violación de los derechos de otros, que inicia desde los 15 años de edad y que se manifiesta por tres o más se los siguientes acontecimientos; 1.- hay un incumplimiento de las normas sociales en lo que respecta a comportamientos legales y se manifiesta por acciones repetidas que son causa de detención. 2.- engaño, que se exterioriza en mentiras repetidas, utiliza sobrenombres - alias además de estafa para placer o beneficio personal. 3.- impulsividad o fracaso para realizar planes con anticipación. 4.- irritabilidad y agresividad, que se exteriorizan con riñas y agresiones físicas en forma repetida. 5.- falta considerable de atención hacia la seguridad de otros y la propia. 6.- constante 
irresponsabilidad que se evidencia por la repetida incapacidad de mantener un comportamiento laboral adecuado y cumplir con obligaciones económicas. 7.falta de culpa y remordimiento, que se muestran mediante indiferencia o racionalización del hecho de haber lastimado, herido o robado a otros. Posteriormente se debe tomar en cuenta B) el sujeto tiene como mínimo 18 años. C) hay evidencia de trastornos de conducta que iniciaron antes de los 15 años. D) el comportamiento antisocial no se ocasiona exclusivamente en el curso de un trastorno bipolar o esquizofrenia.

Varios autores sostienen que los criterios diagnósticos del DSM más que rasgos de personalidad hacen referencia a un listado de conductas de naturaleza antisocial, delictiva y penal (17). Stone realizó una crítica a las criterios de diagnóstico del DSM-IV-TR, por ser estrictamente conductuales (1) .

Mientras que la Organización Mundial de la Salud en el CIE-10 (28) describe que para considerarse a un paciente con trastorno de personalidad antisocial debe cumplir al menos cuatro de los criterios generales de trastorno de la personalidad que se describen enseguida, 1.- cruel despreocupación por los sentimientos de otros e incapacidad de empatía, 2.- manifiesta una actitud persistente de irresponsabilidad y desprecio de las normas, reglas y obligaciones sociales, 3.- Incapacidad para mantener relaciones personales duraderas, 4.- muy poca tolerancia a la frustración, 5.- con un bajo umbral para descargas de agresividad, pudiendo llegar incluso a comportamientos violentos, 6.- incapacidad para sentir culpa y para aprender de las experiencias, particularmente del castigo, 7.- existe una predisposición a culpar a otros u ofrecer racionalizaciones verosímiles de los comportamientos conflictivos. Dentro de los comentarios se menciona además que puede añadirse al cuadro clínico una irritabilidad persistente y la presencia de trastornos de conducta durante la infancia y adolescencia, aunque no son requerimientos necesarios.

Algunos autores mencionan que el TAP es una condición psiquiátrica cuyo esquema radica en manipular y violentar los derechos de otras personas, además de que se incumplen las normas sociales y desacatan la ley. Las personas que padecen este trastorno suelen ser indiferentes a los sentimientos y sobre todo al dolor de los demás y suelen no mostrar remordimiento alguno cuando lastiman a otros así como decisiones imprudentes (29).

Otros autores afirman que lo que realmente caracteriza a este trastorno de personalidad son los comportamientos impulsivos, ya que no permiten detenerse a analizar las consecuencias negativas de dichas conductas, además de que existen déficits en cuanto a la solución de problemas, una notable falta de responsabilidad en aspectos personales y sociales, así como la ausencia de sentimientos de culpa o de amor (30).

\section{Diagnóstico Diferencial}

La comorbilidad entre los trastornos de personalidad es un continuo problema en cuanto al diagnóstico y tratamiento de estos padecimientos, existen de igual forma dificultades en cuanto a la etiología, la jerarquía en la que deben ubicarse los trastornos y el tipo de tratamiento psicológico (31).

El paciente con trastorno antisocial de la personalidad puede confundirse con el paciente paranoide, debido a que en el antisocial se desarrolla una creencia de que es objeto de una persecución deliberada debido a una conspiración contra él, muestra dificultades para controlar la ira, lo que frecuentemente resulta en actos violentos, sin embargo, dentro del TAP la paranoia se considera como un mecanismo defensivo, ya que existen patrones psicodinámicos que estas dos patologías comparten, la diferencia radica en que en el trastorno paranoide el sujeto puede sentir culpa y remordimiento después de mostrarse violento, mientas que el paciente con TAP se muestra indiferente ante el daño generado a otros, además los criterios diagnósticos no deben estar presentes únicamente durante un episodio donde el sujeto lleve a cabo conductas antisociales, durante un brote psicótico, un trastorno del estado de ánimo o en el transcurso de una esquizofrenia (1) .

El paciente con TAP puede confundirse con el narcisista ya que, en primer lugar se debe considerar que según algunos autores como Kernberg el trastorno antisocial es sólo una variante primitiva del trastorno narcisista de la personalidad, así que más que un diagnóstico diferencial se considera que existe cierta comorbilidad entre estas patologías, ya que se ha demostrado que el narcisismo maligno es universal en los pacientes antisociales, es decir en su mayoría los antisociales presentan algunos de los rasgos del narcisismo, entre los que se encuentra que en primera instancia idealizan a sus objetos y posteriormente los desvalorizan, la falta de empatía por lo que se muestra renuente a reconocer o identificarse con los sentimientos y necesidades de los demás, con tendencias a ser explotador en sus relaciones interpersonales; sin embargo existen algunas diferencias, la principal radica en que el antisocial muestra un fracaso para adaptarse a las normas sociales, que lo lleva a la transgresión de la ley, así como la despreocupación, irresponsabilidad, falta de culpa y remordimiento por el daño ejercido a otros, estos criterios son los que diferencian cuando se trata de un paciente con TAP o de un trastorno narcisista de la personalidad (1).

El antisocial a menudo se superpone con el paciente límite, sin embargo en su mayoría son mujeres las que presentan el trastorno límite, mientras que se tienen registros de que el TAP es presentado principalmente por varones, de igual forma ambos comparten la impulsividad y la agresividad excesiva, la diferencia entre estos radica en que el paciente antisocial se caracteriza por relaciones interpersonales superficiales donde no muestra afectividad o interés por el otro, más bien lo utiliza para cumplir sus objetivos y puede dar por concluida la relación en el momento en que no le es útil, mientras que en el paciente límite existen esfuerzos frenéticos por 
evitar el abandono real o imaginario, además de que pueden manifestar intensos sentimientos de culpa debido a este abandono cosa que no acurre con el paciente con TAP (1).

En un estudio realizado con reclusos daneses se comprobó que además de cumplir con los criterios para ser diagnosticados con TAP, presentaban puntuaciones altas en otras patologías como psicosis, trastornos por dependencia y trastornos relacionados con el estrés (6).

Debido a la comorbilidad del TAP se considera al enfoque psicodinámico y la evaluación que éste realiza como una opción que permite evaluar de manera más integral.

\section{Características Clínicas Psicodinámicas del Trastorno Antisocial de la Personalidad}

La perspectiva psicoanalítica permite no sólo enunciar los síntomas sino la descripción de los procesos mentales del sujeto, así mismo permite la descripción de las características clínicas propias de cada patología y de su manifestación particular en cada sujeto.

Respecto a las investigaciones psicoanalíticas sobre el TAP, son escasas las aportaciones que se han realizado a lo largo de la historia, además de que no existe unanimidad en los autores que se han ocupado del estudio de ésta patología, pese a esto la clínica psicoanalítica ha podido precisar algunas características sobre esta patología como: la existencia de una notable debilidad del yo, graves dificultades en cuanto a los mecanismos disociativos que originan una insuficiente discriminación entre los buenos y los malos objetos, así como entre el self y los objetos, estos sujetos presentan un superyó primitivo, hay un predominio de la acción (acting out) por encima del pensamiento debido a un déficit en la simbolización del sentido de realidad, del esquema corporal y el discernimiento heterohomosexual, subordinación del principio de realidad ante el principio del placer de modo que las capacidades del yo se utilizan para liberarse de forma inmediata de la ansiedad generada por la más mínima frustración (32).

Kernberg refiere que los paciente con una estructura borderline, donde se ubica el TAP, presentan una serie de características de personalidad, siendo una de las más importantes la labilidad yoica, que alude a tres elementos; el primero de ellos se refiere a que el paciente presenta una falta de tolerancia a la ansiedad, en segundo lugar existe una incapacidad en el control de impulsos, y finalmente un insuficiente desarrollo de los canales de sublimación (33).

Estos pacientes oscilan entre estados emocionales intensos, pero lábiles; incapacidad para realizar modificaciones a su conducta; ausencia de culpa y remordimiento consciente por el daño infringido a otros; aun cuando pueden mostrarse muy apasionados carecen de una verdadera capacidad para amar, tienden a ser demandantes y exigentes con sus familiares y personas vinculadas de alguna forma con ellos, sin ser recíprocos pues se niegan a brindar cualquier tipo de apoyo cuando otros se lo solicitan, existe en estos pacientes una inadecuada apreciación de sus propias capacidades y posibilidades, ya que siempre se enjuician de forma omnipotente (32).

Las relaciones de objeto en estos pacientes son patológicas, ya que están basadas en la envidia. Se devalúa al objeto como defensa contra la envidia y hay una apropiación de ideas de otros. Son incapaces de depender verdaderamente de una relación con el objeto y empatizar o comprometerse con los otros (26).

Las personalidades antisociales aprenden a pensar lo impensable, sostener lo insostenible, y a postular como cierto lo que saben muy bien que es falso, esto les ha sido introyectado por sus relaciones de objeto primarias (27). Lo anterior deja en claro que no todas las personas que padecen trastorno antisocial se encuentran en las cárceles, sino que muchos de ellos llevan una vida aparentemente normal, sin embargo los rasgos psicopáticos se hacen evidentes en menor medida ya que estos sujetos suelen ser más cautelosos y astutos para evitar ser descubiertos.

Además al manifestar sus afectos, los pacientes con TAP suelen mostrarse irritables y con incapacidad de establecer vínculos afectivos fuertes y duraderos, se muestran apáticos y sin remordimiento o culpa cuando dañan a otros (20). Según el ambiente en el que se encuentre, el sujeto con trastorno antisocial busca mantener el control, esta es una de sus principales motivaciones, ya que no puede establecer relaciones interpersonales, debido a una incapacidad para sentir amor, compasión o solidaridad, por lo que busca el domino de quienes lo rodean (34).

Bromberg (27) plantea que el respeto por la ley representa la resolución de conflicto del sujeto con sus hostilidades inconscientes hacia las figuras de autoridad. Por lo que afirma que la hostilidad y la agresión del paciente con TAP contra la sociedad son una forma de masoquismo moral, que se relaciona con una necesidad inconsciente de castigo.

En la conducta psicopática la meta principal es evitar la tensión que resulta cuando los impulsos no se satisfacen, se evita la ansiedad cuando la frustración es inminente, se protege al yo de sentimientos de inadecuación. No hay un desarrollo de defensas neuróticas adecuadas, por lo que se hace necesario que el paciente escape de la frustración y la ansiedad. (26).

La escisión del yo en los pacientes antisociales, tiene además, la función esencial de proteger al sujeto contra la angustia, por lo que se considera que no es sólo una deficiencia del yo, sino una defensa muy intensa, cuyo objetivo es protegerse de las tempranas relaciones objetales patológicas internalizadas. (35).

Se considera que los paciente con trastorno antisocial recurren a la acción defensiva, es decir, al acting out, para enfrentar las dificultades que tienen respecto a la culpa que se encuentra en el plano psíquico, es decir, es inconsciente, así es como la actuación psicopática 
implica una tentativa de estos sujetos por negar la culpa que les es intolerable, haciéndola recaer sobre los demás, lo que habla de una debilidad del yo para tolerar los sentimientos de culpa, por lo que recurre a la negación y a la identificación proyectiva como mecanismos defensivos que le permiten trasladar esa culpa hacia otros. (27).

\section{Tratamiento}

A lo largo de la historia se han tenido diferentes opiniones respecto al tratamiento que deben recibir los pacientes con trastorno antisocial de la personalidad, sin embargo de manera general se ha considerado que el tratamiento incluye programas multimodales los cuales incluyen aspectos médicos, familiares, psicológicos y comunitarios (36).

Pese a que durante mucho tiempo se ha considerado que el trastorno antisocial de la personalidad no es posible de ser tratado mediante psicoterapia debido a que no se obtenían resultados favorables y por el contrario empeoraba la situación del paciente pues dentro del espacio terapéutico el paciente pudiese desarrollar mayores habilidades de manipulación y control hacia los demás; hay investigaciones que reportan resultados positivos. Estos estudios son sumamente escasos y con serias deficiencias metodológicas pero plantean a la psicoterapia como una opción viable y adecuada para el tratamiento de los psicópatas (37).

Entre estas investigaciones se encuentra la de Salekin, Worley y Grimes, (38) en la que se revisan 16 casos de tratamientos psicoterapéuticos de pacientes con TAP, siendo 8 adultos y 8 adolescentes; se pudo concluir que sólo 3 de los 8 adultos reportan beneficios gracias al tratamiento psicológico, mostrando una reducción moderada de violencia, reducción en conductas de agresión sexual, así como mayor constancia e interés por la psicoterapia, sin embargo, en el caso de los jóvenes, 6 de los 8 participantes mostraron mejora significativa en áreas como reducción de violencia, menor confrontación con las figuras de autoridad, además de una reducción de los rasgos psicopáticos, así como un mejor manejo de conflictos y mayor disciplina.

Por su parte Garrido (37) realizó un meta-análisis basado en 26 estudios de autores que realizaron intervenciones terapéuticas con pacientes antisociales o psicópatas, con el objetivo de determinar cuáles son los factores que pueden influir en la eficacia y eficiencia de los tratamientos aplicados a sujetos que presentan esta patología; los resultados del meta-análisis determinaron que hay algunos factores que intervienen y ayudan a que los pacientes tengan mejora, entre ellos están que el sujeto tenga menos de 30 años de edad, que el tratamientos se aplique por periodos prolongados, que sea aplicado en contextos penitenciarios con sujetos que han cometido un delito y que estos presenten niveles bajos de psicopatía.

Las investigaciones anteriores comprueban que la psicoterapia puede beneficiar a los pacientes con TAP, sobre todo si ésta se ofrece durante la infancia o adolescencia, etapa en la que comienzan a manifestarse los primeros síntomas de la patología, ya que pueden obtenerse resultados mayormente significativos que si se aplica en pacientes adultos que ya tienen una estructura psíquica constituida.

Dentro de los enfoques psicoterapéuticos para el tratamiento de el TAP se encuentran el cognitivoconductual como uno de los más utilizados, ya que se piensa que el sujeto incurre en conductas antisociales y agresivas debido a que está predispuesto a situaciones hostiles, por lo que la Terapia Cognitivo Conductual se enfoca en el desarrollo interpersonal y cognoscitivo de destrezas para solución de problemas, el objetivo es orientar al paciente a mejorar su conducta social, mediante técnicas que incluyen juegos, historias así como varios tipos de rol playing y reforzamiento (21).

Otro tipo de terapia que parece tener resultados favorables en el tratamiento de adolescentes con trastorno disocial o rasgos psicopáticos es la terapia Multisistémica (TMS), que integra aspectos biológicos, psicosociales y psicoeducativos los cuales tienen una ambientación ecológica. La TMS se enfoca en identificar problemas del paciente, generalmente se trata de adolescentes y su familia dentro del contexto comunitario e interviene en un ambiente natural. El objetivo es dotar a los padres de habilidades para la crianza del adolescente, por medio de intervenciones individualizadas y altamente flexibles donde se proporcionan estrategias familiares, individuales y farmacológicas para ayudar al paciente. Además se cuenta con un equipo de apoyo y monitoreo continuo de los objetivos, integrado por psiquiatras, trabajadores sociales, psicólogos y otros profesionales de la salud (21).

Como se ha revisado hasta el momento las intervenciones con pacientes psicopáticos se han basado en supuestos teóricos de que la conducta antisocial es de cierto modo aprendida y se mantiene gracias al déficit en habilidades sociales y cognitivas. Por lo que el objetivo, hablando de forma general, de estos tratamientos es modificar el repertorio de conductas y cogniciones inadecuadas mediante el entrenamiento del paciente en competencias sociales para generalizarlas en los diversos contextos en los que se desenvuelve y aminorar con ello la presencia de conductas psicopáticas (39).

De esta manera la orientación de los tratamientos psicológicos para atender el trastorno antisocial de la personalidad, van dirigidos en su mayoría, a la modificación de conductas y cogniciones del paciente prestando poca atención a procesos psíquicos más profundos, como las instancias psíquicas -ello, yo, superyó-, el tipo de estructura psíquica, el desarrollo psicosexual, las relaciones de objeto, los mecanismos defensivos que emplean estos pacientes, por mencionar algunos y la relación de estos respecto a la patología. 
Si bien son pocos los estudios sobre el enfoque psicoanalítico en el tratamiento de pacientes con trastorno antisocial, las psicoterapias psicodinámicas han mostrado efectividad en tratamientos de pacientes con esquizofrenia, además de mejoras significativas en trastornos como las personalidades antisociales, limítrofes, narcisistas y en general para los trastornos de personalidad y trastornos afectivos (40).

Malan (40) afirma que con las psicoterapias psicodinámicas, se pueden alcanzar cambios significativos en enfermedades mentales que son consideradas como graves y crónicas, incluyendo al trastorno antisocial de la personalidad.

Es importante mencionar que en México se ha implementado un ensayo observacional prospectivo con 19 pacientes diagnosticados dentro de la organización borderline aplicando la Psicoterapia Focalizada en la Trasferencia (PFT) o psicoterapia psicodinámica expresiva, por un periodo de dos años, los resultados que se obtuvieron después de 24 sesiones fueron la desaparición de los criterios de impulsividad, posterior a las 48 sesiones disminuyeron casi por completo los criterios de inestabilidad afectiva y quienes llegaron a las 72 sesiones lograron incrementar en un 70 por ciento su actividad global, sin embargo no se obtuvieron mejoras significativas en cuanto a alteraciones de identidad (30).

La psicoterapia psicodinámica expresiva o Psicoterapia Focalizada en la Transferencia (PFT) se ha indicado para pacientes fronterizos o limítrofes, debido a que se han obtenido mayores resultados que con otros tipos de terapia, sus objetivos apuntan a la disminución de síntomas, promueve un cambio en el paciente respecto a su concepción de sí mismo y que este logre una integración de las representaciones sobre los objetos que han sido escindidos durante las primeras etapas de desarrollo. Propone que la intervención se lleve a cabo por lo menos durante un año, de una a dos veces por semana, dependiendo de las condiciones del paciente, poniendo énfasis en el encuadre y el contrato terapéutico, a fin de establecer claramente las metas y objetivos de la psicoterapia y que estos se tengan siempre presentes tanto por el analista y sobre todo por el paciente (42) .

Además de la psicoterapia psicodinámica expresiva o PFT, algunos autores sugieren que puede emplearse la psicoterapia psicodinámica de apoyo para brindar atención a los pacientes que padecen un trastorno antisocial de la personalidad. El objetivo de la psicoterapia psicodinámica de apoyo dirigida al paciente antisocial consiste en establecer objetivos claros y definidos por ambas partes, es decir, paciente y terapeuta mediante un contrato terapéutico, donde a diferencia de la terapia psicoanalítica, el clínico tiene una gran actividad dentro del espacio terapéutico, siendo este quien dirige la sesión, enfocándola en lo referente a los conflictos cotidianos del paciente que tiene que ver más con situaciones de conducta, es así que no se hace uso de la interpretación de los fenómenos inconscientes del paciente, sin embargo se realiza una explicación al paciente sobre sus necesidades, temores, su intensa necesidad de expresar sentimientos de ira o rabia, esto con el objetivo de que sea capaz de vincular sus estados emocionales con sus acciones y sea capaz de expresar verbalmente sus sentimientos reemplazando el acting out y las conductas autodestructivas (43).

Entre los objetivos primordiales de la psicoterapia de apoyo se encuentran lograr una adaptación respecto a los factores estresantes evitando el insight hacia las fantasías y los deseos inconscientes, el analista trabaja para fortalecer las defensas lo cual facilita la capacidad de adaptación lo que le permite un mejor manejo de conflictos y situaciones estresantes de la vida cotidiana (44).

La psicoterapia de apoyo se enfoca además, en identificar los eventos que son desencadenantes de una posible crisis y también en un primer momento, realiza una evaluación sobre los mecanismos y herramientas psicológicas con las que cuenta el paciente para enfrentar sus conflictos, por lo que este tipo de psicoterapia de enfoque psicodinámico se orienta en el significado subjetivo del evento desencadenante, así como de los antecedentes inconscientes y conscientes de dichos significados y en los mecanismos defensivos que emplea el sujeto para enfrentar las situaciones que le generan un conflicto psíquico (45)

De manera específica en relación al tratamiento psicodinámico de los pacientes con TAP, uno de los objetivos de la psicoterapia de apoyo respecto a las personalidades borderline, donde se ubica el trastorno antisocial de la personalidad, radica en que el paciente logre verbalizar sus emociones, sobre todo la ira o el enojo que se relacionan con la impulsividad y agresión, ya que estos pacientes tienden a expresar su ira con acciones y no de forma verbal, por lo que el terapeuta debe fomentar que el paciente exprese verbalmente sus emociones para que logre con ello la identificación de estas y pueda integrarlas, además se busca que sea capaz de identificar aquellas situaciones que las generan a fin de lograr con el trascurso de las sesiones una regulación emocional y disminuir con ello, conductas impulsivas y de agresión hacia sí mismo y hacia el mundo exterior (45).

Tras esta revisión, se puede concluir que las psicoterapias psicodinámicas son un opción de tratamiento viable para la intervención en el trastorno antisocial de la personalidad ya que no sólo se basa en las conductas sino profundiza en los aspectos psíquicos del paciente y si bien en un primer momento el psicoanálisis no se consideró como una opción viable para el tratamiento de este tipo de trastornos, con las modificaciones a la técnica y el desarrollo de las psicoterapias psicodinámicas plantean un nuevo desarrollo del campos psicoanalítico, tanto a nivel teórico como técnico, que permiten ampliar su campo clínico de acción (46). 


\section{Referencias}

Mackinnon, R. A., Michels, R. \& Buckley, P.J. (2008). Paciente antisocial La entrevista psiquiátrica en la práctica clínica. (325- 353). México: Ars Médica.

Herrera, J. (2015). Uso de la categoría trastorno antisocial de la personalidad como invisibilización de la violencia feminicida. Revista estudios de género. La ventana, 42, 100-128. [Online]. Available from: http://www.redalyc.org/pdf/884/88446716005.pdf [Accessed 20 August 2018].

Borges, G., Medina-Mora, M. A. \& López-Moreno, S. (2004). El papel de la epidemiología en los trastornos mentales. Salud pública de México, 46(5), 451-463. [Online]. Available from: http://www.scielo.org.mx/scielo.php?script=sci_arttext\&pid=S003636342004000500011 [Accessed 20 August 2018].

World Mental Health Consortium. (2007). Lifetime prevalence and age-ofonset distributions of mental disorders in the World Health Organization's World Mental Health Survey Initiative. World Psychiatry, 6(3), 168-176. [Online]. Available from: http://www.ncbi.nlm.nih.gov./pmc/articles/PMC2174588/ [Accessed 20 August 2018].

Rodríguez, J. Kohn, R. \& Aguilar-Gaxiola, S. (2009). Epidemiología de los trastornos mentales en América Latina y el Caribe. Washington: Organización Panamericana de la Salud.

Martínez, T. (2015). Psicopatía, violencia y trayectoria delictiva: análisis de su interacción en muestras penitenciarias. (Tesis doctorado, Universidad complutense de Madrid). [Online]. Available from: http://eprints.ucm.es/33701/1/T36560.pdf [Accessed 20 August 2018].

Benjet, C., Borges, G., Medina-Mora, M. E., Mendez, E., Fleiz, C., Rojas, E. \& Cruz, C. (2009). Diferencias de sexo en la prevalencia y severidad de trastornos psiquiátricos de adolescentes de la Ciudad de México. Salud Mental, 32(2), 155-163. [Online]. Available from: http://www.scielo.org.mx/pdf/sm/v32n2/v32n2a8.pdf [Accessed 20 August 2018].

Vásquez, J. Feria, M. Palacios, L. \& De la Peña, F. (2010). Guía clínica para el trastorno disocial. México: Instituto Nacional de Psiquiatría Ramón de la Fuente.

Instituto Nacional de Psiquiatría Ramón de la Fuente. (2014). El trastorno de la personalidad antisocial. Información clínica, 25(4), 31-33. [Online]. Available from http://www.bic.edilaser.net/es/pdf/2504/04_BIC_Abril_2014_p31 33.pdf [Accessed 20 August 2018].

Kernberg, P. Weiner, Alan. S. \& Bardenstein, Karen. K. (2002). Trastorno antisocial de la personalidad. Trastornos de personalidad en niños y adolescentes. México: Manual Moderno.

Organización Panamericana de la Salud [OPS] \& Organización Mundial de la Salud [OMS]. (2011). Informe sobre el sistema de salud mental en México. México: Biblioteca de la OMS

Organización Mundial de la Salud [OMS]. (2001). Carga de los trastornos mentales y conductuales. Informe sobre la salud en el mundo. Salud mental: nuevos conocimientos, nuevas esperanzas. (19.45). Francia: Biblioteca de la OMS.

Secretaria de Salud. (2016b). Actualización del Programa Institucional de los Servicios de Salud de Hidalgo 2011-2016. [Online]. Available from: http://s-salud.hidalgo.gob.mx/wp-

content/Documentos/Actualizacion\%20de1\%20Programa\%20Institucio nal $\% 20 \mathrm{de} \% 201$ os $\% 20$ Servicios $\% 20 \mathrm{de} \% 20$ Salud $\% 20 \mathrm{de} \% 20 \mathrm{Hidalgo} \%$ 202014.pdf [Accessed 20 August 2018].

Larrieta, C. (23 de noviembre 2016). Hidalgo referente en atención en salud. Diario Plaza Juárez. [Online]. Available from: http://www.elvistobueno.com.mx/2016/11/hidalgo-referente-enatencion-de-salud-mental/ [Accessed 20 August 2018]

Vázquez, P. \& Gómez, R. A. (2015). Funcionalidad familiar y patología dual en usuarios residenciales del Centro Estatal para la Atención
Integral de las Adicciones. Universidad Autónoma del Estado de Hidalgo.[Online]. Availablefrom:https://repository.uaeh.edu.mx/revist as/index.php/ICSA/article/view/853/851 [Accessed 20 August 2018].

Azaola, E. (2015). Diagnóstico de los adolescentes que cometen delitos graves en México. México: UNICEF.

Caballo, V. (2004). El trastorno antisocial de la personalidad. Manual de trastornos de la personalidad. Descripción, evaluación y tratamiento. España: Síntesis.

American Psychiatric Association., Kupfer, D. J., Regier, D. A., Arango López, C., Ayuso-Mateos, J. L., Vieta Pascual, E., \& Bagney Lifante, A. (2014). DSM-5: Manual diagnóstico y estadístico de los trastornos mentales (5a ed.). Madrid [etc.]: Editorial Médica Panamericana.

Estrada, C. Chan, E. C. \& Rodríguez, F. J. (2011).Comportamiento antisocial delictivo: teorías y modelos. Delito e intervención social. Una propuesta para la intervención profesional. México: UDG.

Arias, G. N. \& Ostrosky-Solis, F. (2008). Neuropsicología de la violencia y su clasificación. Revista de neuropsicología neuropsiquiatría y neurociencias, 8(1), 95-114. [Online]. Available from: https://dialnet.unirioja.es/servlet/articulo?codigo=3987506 [Accessed 20 August 2018].

De la Peña, F. R. (2003). Tratamiento multisistémico en adolescentes con trastorno disocial. Dep. de Psicología, Médica y Psiquiatría de la UNAM, 45(1), 124-131. [Online]. Available from: http://scielo.unam.mx/pdf/spm/v45s1/15453.pdf [Accessed 20 August 2018].

Justicia, F., Benítez, J. L., Pichardo, M. C., Fernández, E., García, T. \& Fernández, M. (2006). Aproximación a un nuevo modelo explicativo de la conducta antisocial. Revista electrónica de investigación psicoeducativa, 9(4), 1696-2095. [Online]. Available from: http://www.investigacion-

psicopedagogica.org/revista/articulos/9/espannol/Art_9_117.pdf [Accessed 20 August 2018].

Hikal, W. (2012). Estudio de la personalidad antisocial durante el diagnóstico clínico. Revista electrónica de psicología Iztacala, 15(3), 871-886. [Online]. Available from: http://www.iztacala.unam.mx/carreras/psicologia/psiclin/vol15num3/V ol15No3Art5.pdf [Accessed 20 August 2018].

Cabanach, A. Valle, R. Arce, F. \& Fariña, F. (2011). Comportamientos antisociales y delictivos en menores, efectos de una socialización diferencial. Psicología y Salud. Coruña: Psicoeduca.

Fernández, C. \& Rodríguez, L. (2013). Tratamiento psicoanalítico de los trastornos de personalidad. Revista Acción Psicológica, 10(1), 57-64. [Online]. Available from: http://scielo.isciii.es/pdf/acp/v10n1/monografico6.pdf [Accessed 20 August 2018].

Ortiz, E. C. (2000). Estudio de las personalidades y los rasgos de carácter psicopáticos. (Tesis maestría. Centro ELEIA de actividades psicológicas).

Mollo, J. P. (2010). Psicoanálisis y criminología. Buenos Aires: PAIDÓS.

Organización Mundial de la Salud. [OMS]. (1992). Décima revisión de la clasificación internacional de las enfermedades. [CIE-10]. Trastornos mentales y del comportamiento. Madrid. Ed. Méditor.

Alvarado, I. Rosario, LL. \& García, N. (2013). Trastorno antisocial de la personalidad en personas institucionalizadas en Puerto Rico: Estudio de casos. Revista puertorriqueña de psicología, 25(1), 062077.[Online]. Available from: http://web.a.ebscohost.com/ehost/pdfviewer/pdfviewer?sid=069b1643ad7f-49df-819b-39580bf71bd5\%40sessionmgr4009\&vid=2\&hid=4109 [Accessed 20 August 2018].

López, M. J. \& Núñez, M. C. (2009). Psicopatía versus trastorno antisocial de la personalidad. Reic, 1(7), 1-14. [Online]. Available from: http://www.criminologia.net/pdf/reic/ano7-2009/a72009art1.pdf [Accessed 20 August 2018]. 
Oldham, J. M. \& Fowler, J. C. (2013). Trastornos de personalidad I. Estados Unidos: American Psychiatric Association.

Coderch, J. (1974). La estructura de las personalidades psicopáticas desde el punto de vista del psicoanálisis. Rev. Psiquiatría facultad de medicina Barna, 2(3), 155-166. [Online]. Available from: http://www.psicoterapiarelacional.es/Portals/0/Documentacion/JCoder ch/Coderch_1974_La\%20estructura\%20de\%201as\%20personalidades \%20Psicopaticas.pdf [Accessed 20 August 2018].

Fossa, P. (2010). Organización limítrofe de la personalidad. Revista de psicología GEPU, 1(1), 32-52.

Pazos, Y. (2014). Relación de la modernidad con el incremento del trastorno de personalidad antisocial en la población norteamericana: caso de estudio Ted Burndy. (Tesis licenciatura). [Online]. Available from: http://132.248.9.195/ptd2014/diciembre/305331520/Index.html [Accessed 20 August 2018].

Bateman, A. \& Fonagy, P. (2005). Psicoterapia para el trastorno límite de la personalidad. México: Editorial Universitaria.

Vásques M.J., Feria M, Palacios L \& De la Peña F. (2010). Guía clínica para el trastorno disocial. Ed. Shoshana.

Garrido, V. (2002). El tratamiento del psicópata. Psicothema, 14(1), 181189. [Online]. Available from: http://www.psicothema.com/pdf/3489.pdf [Accessed 20 August 2018].

Salekin, R. Worley, C. \& Grimes, R. D. (2010). Treatment of psychopathy: A review and brief introduction to the mental model mpproach for psychopathy. Behavioral Sciences, 28(2), 235-266. [Online]. Available from:

http://onlinelibrary.wiley.com/doi/10.1002/bsl.928/epdf?r3_referer=wo $1 \&$ tracking_action=preview_click\&show_checkout=1\&purchase_refer rer=www.google.com.mx\&purchase_site_license=LICENSE_DENIE D [Accessed 20 August 2018].

Silva, A. (2003). Conducta antisocial: un enfoque psicológico. México: Pax.

Gutiérrez, M. \& Villamil, L. J. (2015). La psicoterapia de orientación psicodinámica en el tratamiento integral de pacientes con esquizofrenia y sus familias. Clínica Contemporánea, 6(2), 69-83.

Hales, R. E. Yudofsky, S. C. \& Talbott, J. A. (1996). Tratado de psiquiatría. España: Ancora S.A.

Pérez, L. (2011). Trastorno límite de la personalidad (borderline). Psicoterapia focalizada en transferencia. Revista de psiquiatría Uruguay, 75 (1), 20-25

Díaz, J (2001). Revisión de tratamientos psicoterapéuticos en pacientes con trastornos borderline de la personalidad. Rev Asoc. Neuropsiq, 21(78), 51-70.

Gabbard, G. O. (2002). Psiquiatría psicodinámica en la práctica clínica. Argentina: Médica Panamericana.

Martínez, C. (2005). Intervención en crisis en pacientes con trastorno de personalidad limítrofe. Revista Interamericana de Psicología, 39(1), 151-158.

Caparrós, N. (2007). Perspectiva psicoanalítica del tratamiento de los trastornos de personalidad. Clínica y salud, 18(3), 363-380. 